\title{
Too much mammography
}

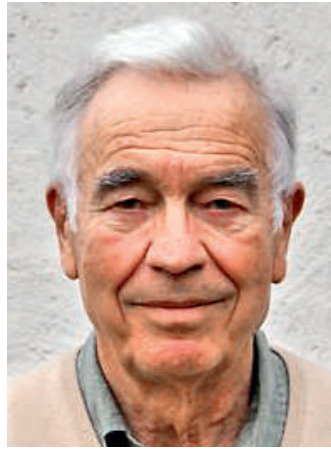

Hans Stalder
«Too much mammography» lautet der Titel eines Editorials im British Medical Journal vom 11. Februar 2014 [1], das sich auf einen Artikel in derselben Ausgabe bezieht. Es geht dabei um eine neue, sehr umfassend angelegte, prospektive und randomisierte Studie, in der die Wirkung einer systematischen Mammographie mit einer Kontrollgruppe verglichen wird [2]. Die Studie zeigt nicht nur keine Wirkung auf die Mortalität, sondern einmal mehr das Problem der Überdiagnose (overdiagnosis) von $22 \%$. Zu viele Mammographien - zu dieser Schlussfolgerung gelangt auch der vor Erscheinen des genannten Artikels verfasste Bericht des Swiss Medical Boards (SMB) [3].

\section{«Die Entscheidung von Frau X ist sicherlich abhängig von unseren Überzeugungen.»}

Die Reaktionen auf die Analyse des SMB liessen nicht lange auf sich warten: «Hört auf, mit der Brust der Frau zu spielen», so eine Professorin aus der Präventivmedizin in der französischsprachigen Presse [4], und ein neu ins Amt gewählter Staatsrat vermutet sogar Machenschaften der Deutschschweiz hinter dem Bericht des SMB [4]. Allerdings gibt es auch differenziertere Kommentare in der schweizerischen [5] und sogar in der ausländischen [6] Presse. Einige sehen die Schweiz gar in einer Vorreiterrolle.

Was mich betrifft, lässt mich der in der Schweizerischen Ärztezeitung veröffentlichte Vorschlag [7] «Die Frauen sollen entscheiden» aufhorchen. Solche Vorschläge sind häufig zu hören - auch vom SMB! -, wenn in einer medizinischen Thematik keine klaren Schlüsse gezogen werden können. Ich habe meine Zweifel angesichts solcher Vorschläge, denn wer gute Entscheidungen treffen will, muss gut informiert sein. Und die in dieser Nummer und in der Presse veröffentlichten Kommentare lassen mich zweifeln, ob diese Informationen immer objektiv sind. Nehmen wir ein Beispiel: Frau X, 50 Jahre, Kassiererin im Supermarkt geht zu ihrem Arzt, unentschieden, ob sie eine Mammographie machen lassen soll oder nicht. Wie wird sie entscheiden?

Stellen wir uns die zwei folgenden Szenarien vor:

1. Der Arzt findet die Schlussfolgerungen des SMB «skandalös» [8] und sagt: «Sie müssen unbedingt eine Mammographie durchführen lassen!» «Warum?» «Brustkrebs ist die häufigste Krebsart bei der Frau. Wir müssen etwas tun! Mit einer Mammo- graphie reduzieren Sie Ihr Risiko, daran zu sterben, um 20\%.»

2. Der Arzt, der gleicher Meinung ist wie das SMB: «Ich rate Ihnen von einer Mammographie ab.» «Warum?» «Bei Frauen ist Brustkrebs zwar die häufigste Krebsart. Jedoch wenn man bei 1000 Frauen über einen Zeitraum von 10 Jahren Mammographien durchführt, sterben zwar maximal 2 nicht am Brustkrebs, jedoch an etwas anderem. Und ohne von falsch positiven Testergebnissen reden zu wollen, besteht ein Risiko von 20\%, dass es bei einem durch die Mammographie entdeckten Krebs in der Folge zu unnötigen Behandlungen kommt.»

Und die Situation könnte noch komplizierter werden: Wer kommt für die Kosten auf, wenn die Frau aus Szenario 1 beschliesst, Mammographien durchführen zu lassen? Ist es richtig, dass die Grundversicherung zahlt, wenn die Schlussfolgerungen des SMB akzeptiert werden? Und wenn die Frau aus Szenario 2 nach drei Jahren an Brustkrebs erkrankt, wird sich da nicht der Arzt Vorwürfe machen und vielleicht sogar der Fahrlässigkeit beschuldigt werden, wie das in Deutschland jüngst der Fall war [9]?

\section{«Wir brauchen eine Stelle, die versucht, wissenschaftliche, unabhängige und neutrale Schlussfolgerungen zu ziehen.»}

Die Entscheidung von Frau X wird sicherlich abhängig sein von unseren Überzeugungen. Daher sollten wir so objektiv wie möglich bleiben. Da dies jedoch derart schwierig ist, brauchen wir eine Stelle, die versucht, wissenschaftliche, unabhängige und neutrale Schlussfolgerungen zu ziehen. Dies ist genau die Aufgabe des SMB. Anstatt das Board mit häufig voreingenommen Argumenten zu kritisieren, sollten wir es lieber ermutigen, seine Arbeit fortzusetzen (und vielleicht noch besser zu machen) und hoffen, dass die Politiker sich nicht durch den Druck gewisser Interessengruppen beeinflussen lassen.

Hans Stalder*

* Prof. Dr. med. Hans Stalder, Facharzt für Innere Medizin, Redaktionsmitglied, ist ehemaliger Direktor der Policlinique de Médecine und des Département de Médecine communautaire des Hôpitaux Universitaires de Genève. 